\title{
ASO Author Reflections: Incomplete Cytoreduction Prediction Model for Pseudomyxoma Peritonei
}

\author{
Mingjian Bai, $\mathrm{MD}^{1,2,3}$, Hongbin $\mathrm{Xu}, \mathrm{MD}^{4}$, and Man Zhang, $\mathrm{MD}^{1,2,3}$ \\ ${ }^{1}$ Clinical Laboratory Medicine, Peking University Ninth School of Clinical Medicine, Beijing, China; ${ }^{2}$ Clinical Laboratory \\ Medicine, Beijing Shijitan Hospital, Capital Medical University, Beijing, China; ${ }^{3}$ Beijing Key Laboratory of Urinary \\ Cellular Molecular Diagnostics, Beijing, China; ${ }^{4}$ Department of Myxoma, Aerospace Center Hospital, Beijing, China
}

\section{PAST}

Pseudomyxoma peritonei (PMP) is a rare disease. ${ }^{1}$ The completeness of cytoreduction is an important prognostic factor for PMP because patients who experienced complete cytoreductive surgery (CCRS) had an 5-year survival rate of $85 \%$ compared with those who had incomplete cytoreduction (IC). ${ }^{2}$ To date, no nomograms have been established to predict incomplete cytoreduction (IC) for patients with PMP.

\section{PRESENT}

The current study developed a nomogram to predict IC for PMP patients. The study enrolled 144 PMP patients between 1 June 2013 and 22 November 2019. Logistic regression modeling identified four factors (sex, disease duration, anemia, and carbohydrate antigen 19-9 [CA 19-9]) that independently predict IC for PMP patients. The study then established a nomogram predicting IC, which demonstrated good predictive accuracy (Cindex, $0.837 ; 95 \%$ confidence interval, $0.764-0.894){ }^{3}$

\section{FUTURE}

A former study found that an experienced radiologist could assess the total peritoneal cancer index (PCI) correctly on both computed tomography (CT) and magnetic resonance imaging (MRI) scans. ${ }^{4}$ The PCI is used to reflect

(C) Society of Surgical Oncology 2021

First Received: 24 August 2021

Accepted: 4 September 2021

Published Online: 21 September 2021

M. Zhang, MD

e-mail: zhangman@bjsjth.cn tumor burden for PMP, and a higher PCI always denotes unresectable disease for PMP. Therefore, the PMP center requires not only experienced surgeons, but also experienced radiologists. In the future, the CT or MRI-PCI will be assessed in the prediction model. Finally, prospective and multi-institutional research should be conducted for external validation aimed at accurate prediction of cytoreducibility among PMP patients to establish preoperative plans.

DISCLOSURE There are no conflict of interest.

\section{REFERENCES}

1. Mittal R, Chandramohan A, Moran B. Pseudomyxoma peritonei: natural history and treatment. Int J Hyperthermia. 2017;5:511-9.

2. Chua TC, Moran BJ, Sugarbaker PH, et al. Early- and long-term outcome data of patients with pseudomyxoma peritonei from appendiceal origin treated by a strategy of cytoreductive surgery and hyperthermic intraperitoneal chemotherapy. J Clin Oncol. 2012;20:2449-56.

3. Mingjian B, Shilong W, Guowei L, et al. Nomogram to predict incomplete cytoreduction for pseudomyxoma peritonei. Ann Surg Oncol. 2021. https://doi.org/10.1245/s10434-021-10725-4.

4. Torkzad MR, Casta N, Bergman A, Ahlstrom H, Pahlman L, Mahteme H. Comparison between MRI and CT in prediction of peritoneal carcinomatosis index (PCI) in patients undergoing cytoreductive surgery in relation to the experience of the radiologist. J Surg Oncol. 2015;6:746-51.

Publisher's Note Springer Nature remains neutral with regard to jurisdictional claims in published maps and institutional affiliations. 